\title{
KERNFORSCHUNGSZENTRUM
}

\section{KARLSRU HE}

Institut für Neutronenphysik und Reaktortechnik

Thermodiffusion im System $\mathrm{UO}_{2}-\mathrm{CeO}_{2}$

H. Beisswenger, M. Bober, G. Schumacher

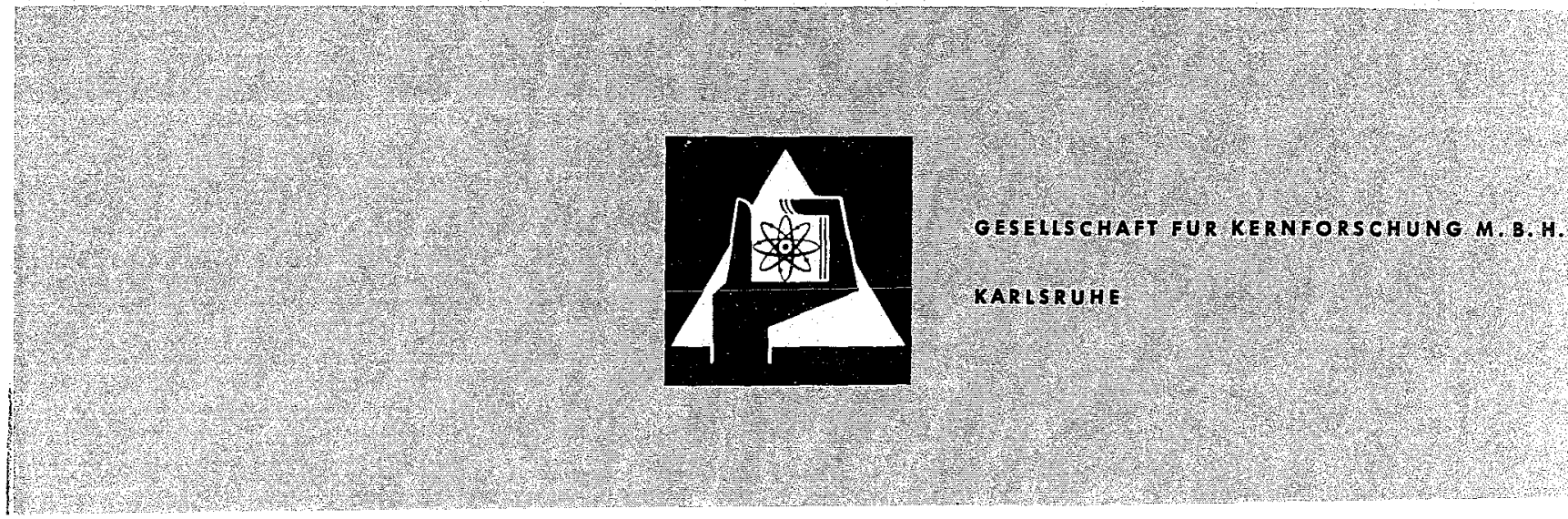




\title{
THERMODIFFUSION IM SYSTEM $\mathrm{UO}_{2}-\mathrm{CeO}_{2}$
}

\author{
H. BEISSWENGER ${ }^{\dagger}$, M. BOBER und G. SCHUMACHER
}

Institut für Neutronenphysik und Reaktortechnik, Kernforschungszentrum Karlsruhe, Deutschland

Eingegangen am 14. Juli 1966

In der vorliegenden Arbeit wird die Thermodiffusion von $\mathrm{CeO}_{2}$ in $\mathrm{UO}_{2}$ untersucht. Es wird eine Anlage beschrieben, in der zylindrische Proben in einem Temperaturgradienten zwischen 1400 und $2200^{\circ} \mathrm{C}$ geglüht werden können. Die $\mathrm{CeO}_{2}$-Konzentration in den verwendeten Proben betrug 3.7, 5.6 und 13.3 Gew \%. Die $\mathrm{CeO}_{2}$-Verteilung nach der Glühung im Temperaturfeld wurde mit der Mikrosonde gemessen. Als wichtigstes Ergebnis wurde festgestellt, dass das $\mathrm{CeO}_{2}$ durch Thermodiffusion in Richtung des positiven Temperaturgradienten wandert. Im unteren Temperaturbereich nimmt daher die $\mathrm{CeO}_{2}$-Konzentration ab und im oberen Temperaturbereich zu. Nach einer Glühzeit von einigen Tagen stellte sich ein Konzentrationsminimum bei einer Temperatur von etwa $1700^{\circ} \mathrm{C}$ und ein Maximum bei etwa $2000^{\circ} \mathrm{C}$ ein. Am oberen Ende der Probe nahm die Konzentration wegen einer Abgabe von $\mathrm{CeO}_{2}$ an die Umgebung wieder ab. Der Konzentrationsverlauf konnte mit Hilfe der elektronischen Rechenmaschine IBM 7070 berechnet werden. Die Rechnung ergab bei $2050^{\circ} \mathrm{K}$ und 13.3 Gew $\% \mathrm{CeO}_{2}$ einen

Diffusionskoeffizienten ' $\quad D=5.4 \times 10^{-8} \mathrm{~cm}^{2} / \mathrm{sec}$, Thermodiffusionskoeffizienten

$D^{\prime}=1.6 \times 10^{-10} \mathrm{~cm}^{2} / \mathrm{sec} \cdot{ }^{\circ} \mathrm{K}$,

Aktivierungsenergie

$$
Q=110 \mathrm{kcal} / \mathrm{Mol} \text {. }
$$

Investigations of thermal diffusion of $\mathrm{CeO}_{2}$ in $\mathrm{UO}_{2}$ are reported. An equipment is described in which cylindrical samples can be annealed in a temperature gradient between $1400^{\circ} \mathrm{C}$ and $2200^{\circ} \mathrm{C}$. The $\mathrm{CeO}_{2}$ concentrations in the used samples were $3.7,5.6$ and $13.3 \mathrm{wt} \%$. The distribution of $\mathrm{CeO}_{2}$ after the annealing procedure in a temperature gradient was analysed by means of an electron microprobe unit.

As the most important result it was established that the $\mathrm{CeO}_{2}$ migrates to the higher temperature by a thermal diffusion process. Thus, the $\mathrm{CeO}_{2}$-concentration decreases in the lower temperature range and increases in the higher temperature range. After annealing some days a minimum of $\mathrm{CeO}_{2}$-eoncentration at a temperature of $1700^{\circ} \mathrm{C}$ and a maximum at $2000^{\circ} \mathrm{C}$ was achieved. At the upper end of the sample the $\mathrm{CeO}_{2}$-concentration decreased because of a $\mathrm{CeO}_{2}$ release to the surrounding volume.

By means of an electronic computer IBM 7070 it was possible to calculate the concentration course. The results of the calculations, at a concentration of $13.3 \mathrm{wt} \% \mathrm{CeO}_{2}$ and a temperature of $2050^{\circ} \mathrm{C}$, were:

Diffusion coefficient $\quad D=5.4 \times 10^{-8} \mathrm{~cm}^{2} / \mathrm{sec}$, Thermal diffusion coefficient

$$
\begin{array}{ll} 
& D^{\prime}=1.6 \times 10^{-10} \mathrm{~cm}^{2} / \mathrm{sec} \cdot{ }^{\circ} \mathrm{K}, \\
\text { Activation energy } & Q=110 \mathrm{kcal} / \mathrm{mol} .
\end{array}
$$

Dans le travail précédent fut étudiée la diffusion thermique de $\mathrm{CeO}_{2}$ dans $\mathrm{UO}_{2}$. Dans ce travail est décrit un dispositif dans lequel des échantillons cylindriques peuvent être chauffés entre 1400 et $2200^{\circ} \mathrm{C}$ dans un gradient de température. La concentration en $\mathrm{CeO}_{2}$ dans les échantillons utilisés était de $3,7,5,6$ et $13,3 \%$ en poids. La répartition de $\mathrm{CeO}_{2}$ après recuit dans le gradient de températures fut mesurée à la microsonde électronique.

Comme résultat le plus important, il fut constaté que l'oxyde de cérium $\mathrm{CeO}_{2}$ se déplaçait par la diffusion thermique dans la direction du gradient de température positif. Dans le domaine des plus basses températures la concentration en $\mathrm{CeO}_{2}$ diminue et augmente dans le domaine des plus hautes températures. Après une durée de recuit de quelques jours, un minimum de concentration s'établit pour une température d'environ $1700^{\circ} \mathrm{C}$ tandis qu'un maximum apparaît pour $2000^{\circ} \mathrm{C}$ environ. A l'extrémité supérieure de l'échantillon, la concentration diminuait à cause d'un départ de $\mathrm{CeO}_{2}$ dans l'atmosphère.

La répartition de la concentration a pu être calculée à l'aide d'une machine à calculer électronique IBM 7070. Le calcul a donné pour $2050^{\circ} \mathrm{C}$ et $13,3 \%$ en poids de $\mathrm{CeO}_{2}$ les résultats suivants:

$$
\begin{aligned}
& \text { Coefficient de diffusion } D=5,4 \times 10^{-8} \mathrm{~cm}^{2} / \mathrm{sec} \\
& \text { Coefficient de diffusion thermique } \\
& D^{\prime}=1,6 \times 10^{-10} \mathrm{~cm}^{2} / \mathrm{sec} \cdot{ }^{\circ} \mathrm{K}, \\
& \text { Energie d'activation } Q=110 \mathrm{kcal} / \mathrm{mol} \text {. }
\end{aligned}
$$

† Delegiert von der Firma NUKEM, Nuklear-Chemie und -Metallurgie GmbH., Wolfgang bei Hanau/Main. 


\section{Einleitung}

Die Thermodiffusion in kondensierten Phasen und speziell im Festkörper hat in der letzten Zeit an Bedeutung und Interesse gewonnen. Eine kinetische Theorie der Thermodiffusion für einfache Strukturen wurde von Wirtz ${ }^{1}$ ) aufgestellt, und neuerdings ist eine umfassende Darstellung der Theorie von de Groot und Mazur ${ }^{2}$ ) wiedergegeben worden. In letzter Zeit wurden auch auf dem experimentellen Gebiet weitere Fortschritte erzielt 3,4 ).

Die Untersuchungen wurden jedoch nur an Systemen, die experimentell verhältnismässig leicht zugänglich sind, durchgeführt. In unserem Falle sollte mit der Untersuchung des Verhaltens von $\mathrm{CeO}_{2}$ in $\mathrm{UO}_{2}$ im Temperaturbereich zwischen 1400 und $2200^{\circ} \mathrm{C}$ ein Problem mit praktischer Bedeutung aufgegriffen werden.

In einem Reaktor-Brennelement bestehen unter Betriebsbedingungen hohe Temperaturdifferenzen zwischen der Stabachse und der Peripherie der Elemente. In diesem Temperaturgradienten, der über lange Zeit bestehen bleibt, können Konzentrationsverschiebungen von Spaltprodukten und Plutonium aufgrund der Thermodiffusion auftreten. Um die Thermodiffusion von $\mathrm{PuO}_{2}$ in $\mathrm{UO}_{2}$ untersuchen $\mathrm{zu}$ können, wurde zunächst mit dem System $\mathrm{UO}_{2}-\mathrm{CeO}_{2}$ die Experimentiertechnik erprobt. $\mathrm{Da} C \mathrm{Ce}$ ein wichtiger Vertreter der seltenen Erden unter den Spaltprodukten ist, liefern die Untersuchungen zugleich Aussagen über das Verhaiten dieser Gruppe der Spaltprodukte.

Solche Konzentrationsverschiebungen von Spaltprodukten in Brennelementen wurden von Bates et al. ${ }^{5-7}$ ) und von Oi et al. ${ }^{8,9}$ ) auch schon beobachtet. Die beiden Gruppen haben an bestrahlten Proben im wesentlichen mit autoradiographischen und aktivierungsanalytischen Methoden gearbeitet. Um die an bestrahlten Proben beobachteten Effekte im einzelnen deuten zu können, erschien es zweckmässig, an $\mathrm{UO}_{2}$ mit entsprechenden Beimengungen ausserhalb des Reaktors im Temperaturgradienten Untersuchungen vorzunehmen.

Die vorliegenden Untersuchungen der Thermodiffusion von $\mathrm{CeO}_{2}$ in $\mathrm{UO}_{2}$ zeigen die wesent- lichen Effekte. In weiteren Experimenten sollen diese Untersuchungen systematisch vervollständigt werden. Gleichzeitig ist beabsichtigt, auch das Verhalten anderer Beimengungen, insbesondere das von Plutonium, zu studieren.

\section{Versuchsanordnung}

\subsection{AuswahL Des Systemis}

Um einen Temperaturgradienten in einem Körper zu erzeugen, gibt es zwei bevorzugte Anordnungen:

a. Ein Hohlzylinder wird mit einem axial angeordneten Heizstab an der Innenseite geheizt und an der äusseren Mantelfläche gekühlt. Der Temperaturgradient verläuft in radialer Richtung.

b. Ein zylindrischer Körper wird an einer Stirnfläche geheizt und an der anderen gekühlt. Der Temperaturgradient verläuft dann parallel zur Zylinderachse.

$\mathrm{Zu}$ a) Die Anordnung mit dem innengeheizten Hohlzylinder hat den Vorteil, dass sie verhältnismässig einfach zu bauen ist.

$\mathrm{Zu} \mathrm{b)} \mathrm{Die} \mathrm{Anordnung} \mathrm{mit} \mathrm{dem} \mathrm{axialen}$ Temperaturgradienten hat den Vorteil, dass der Temperaturgradient über die ganze Probe etwa konstant gehalten werden kann. Ein weiterer Vorteil ist die Möglichkeit, die Probe in einen Behälter einzuschliessen und in diesem Behälter eine definierte Gasatmosphäre aufrechtzuhalten. Die Entscheidung fiel deshalb zugunsten der Anordnung mit axialem Temperaturgradienten.

\subsection{Beschreibung der Verstehs- ANORDNUNG}

Die verwendete Versuchsanordnung ist in Fig. 1 dargestellt. Die Probe befindet sich in einem Probebehälter (5), dessen oberer Teil und dessen Deckel durch Strahlung erhitzt werden. Das Heizelement (2) besteht aus einem Wolframrohr und ist von Abschirmungen (3) umgeben. Auf der unteren Seite der Probe wird die Wärme über den Probeträger (4) und den Probebehälter, die an ihrem unteren Ende gut wärmeleitend mit dem wassergekühlten Halter (6) verbunden sind, abgeleitet. Der Probeträger 


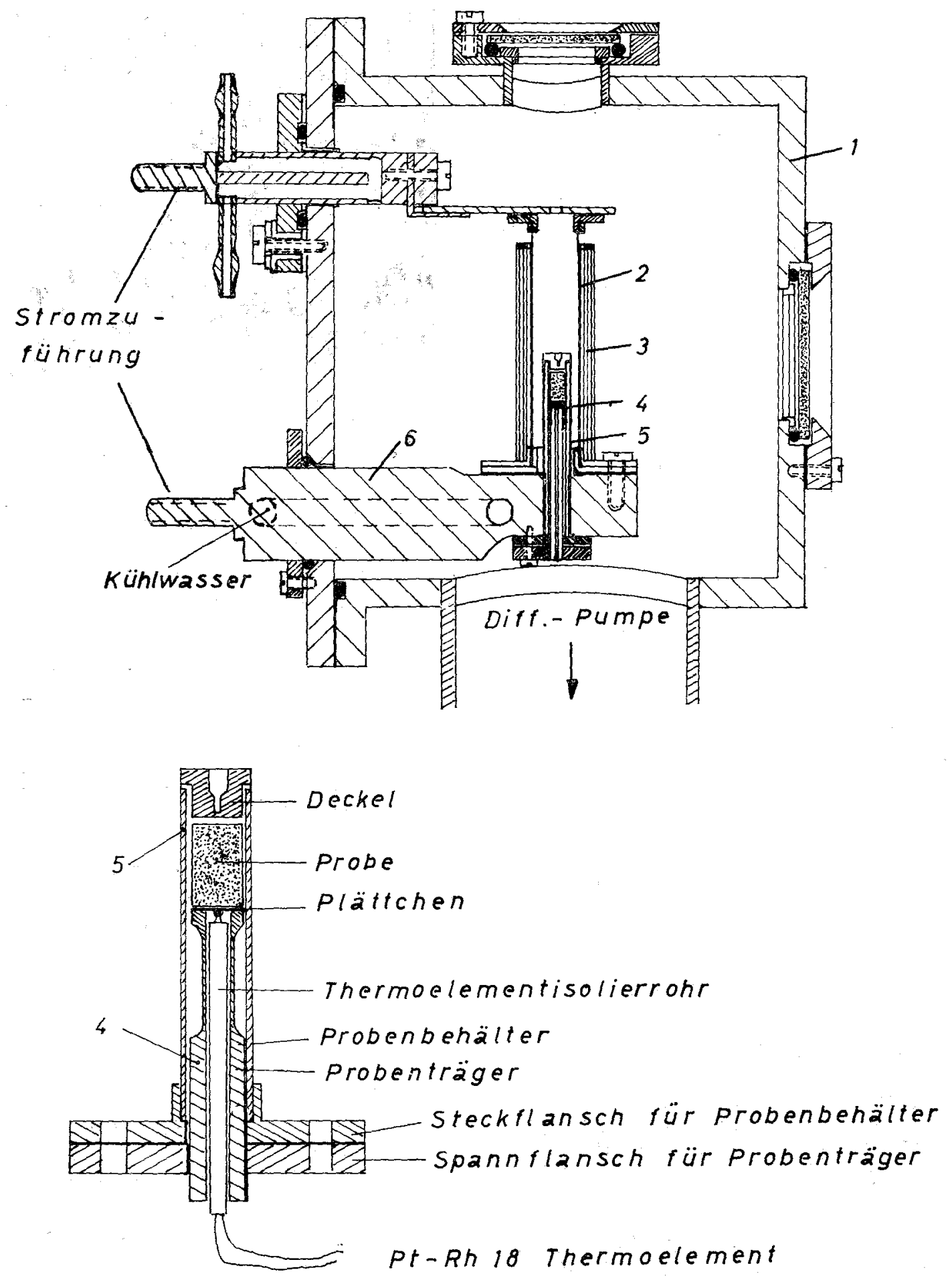

Fig. 1. Versuchsanordnung. 
ist in der Höhe verschiebbar. Sowohl der Probebehälter als auch der Probeträger sind aus hochschmelzenden Metallen gefertigt.

Die Temperatur wird sowohl am Deckel als auch an der Auflage der Probe gemessen. Im Deckel ist hierzu eine Bohrung angebracht, in der mit Hilfe eines optischen Pyrometers die Temperatur in der Nähe der unteren Stirnfläche des Deckels gemessen werden kann. Die untere Seite der Probe sitzt auf einem Plättchen auf, an das ein Pt-Rh-18-Thermoelement angeschweisst ist, mit dem Temperaturen bis zu $1800^{\circ} \mathrm{C}$ gemessen werden können. Der Temperaturverlauf in der Probe wurde durch Eichmessungen, die sowohl mit Thermoelementen als auch mit optischen Pyrometern ausgeführt wurden, ermittelt.

\section{Durchführung der Versuche}

\subsection{Probenhetrstellung}

Die Proben, Pellets mit 6 und $8 \mathrm{~mm}$ Durchmesser und einer Länge von etwa $10 \mathrm{~mm}$, wurden von der Firma NUKEM geliefert. Das Ausgangsmaterial für die Proben war einmal eine mechanische Mischung der beiden Pulver, zum anderen eine über eine gemeinsame Fällung hergestellte Mischung.

Eine gemeinsame Fällung mit 13.3 Gew \% $\mathrm{CeO}_{2}$ war das Ausgangsmaterial für die Proben 1-2. Sie wurden bei $1500^{\circ} \mathrm{C}$ in Wasserstoff 2 Stunden gesintert und erreichten dabei eine

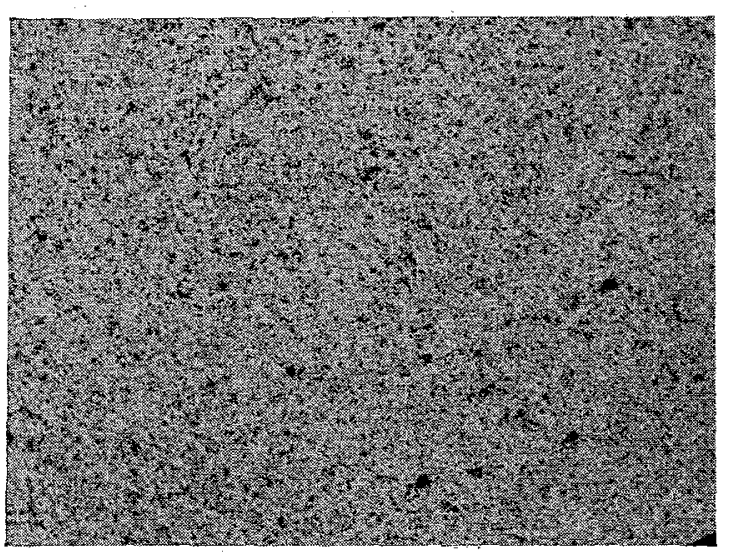

Fig. 2. Gefügebild von unbehandelten Proben. Aus gemeinsamer Fällung mit 13.3 Gew $\% \mathrm{CeO}_{2}$ hergestellte Probe. $\times 100$

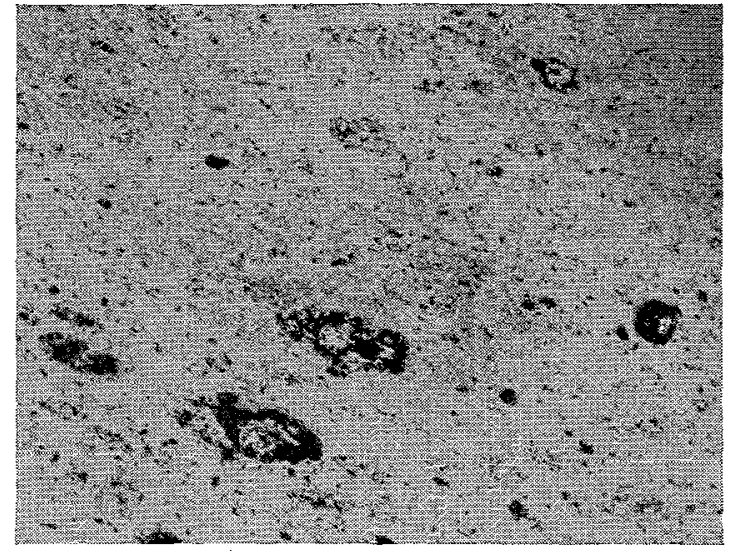

Fig. 3. Gefügebild von unbehandelten Proben. Aus Pulvermischung mit 3.7 Gew $\% \mathrm{CeO}_{2}$ hergestellte Probe. $\times 100$

Dichte von $9 \mathrm{~g} / \mathrm{cm}^{3}$. Das Schliffbild in Fig. 2 zeigt, dass keine $\mathrm{CeO}_{2}$-Agglomerate vorhanden sind und ein feines Korngefüge vorliegt. Probe 3 wurde nach dem gleichen Verfahren wie die Proben 1 und 2 hergestellt. Sie enthielt 5.6 Gew $\% \mathrm{CeO}_{2}$ und ihre Dichte betrug $9.8 \mathrm{~g} / \mathrm{cm}^{3}$. Aus einer mechanischen Mischung mit 3.7 Gew $\% \mathrm{CeO}_{2}$ wurde die Probe 4 hergestellt. Sie wurde bei $1650^{\circ} \mathrm{C}$ in Wasserstoffatmosphäre 2 Stunden gesintert und erreichte eine Dichte von $10 \mathrm{~g} / \mathrm{cm}^{3}$. In Fig. 3 ist das Gefüge dieser Probe in einem Schliffbild dargestellt. An den $\mathrm{CeO}_{2}$ Agglomeraten, die bis zu $100 \mu \mathrm{m}$ gross sind, ist zu erkennen, dass es sich um eine verhältnismässig grobe Pulvermischung handelt. Die Sinterzeit hat nicht ausgereicht, um diese $\mathrm{CeO}_{2}$ Inseln aufzulösen.

\subsection{VERSUCHSABLAUF}

Vor Beginn der Versuche wurde der Temperaturverlauf in einer Eichprobe ermittelt. Als Eichprobe diente ein zylindrisches $\mathrm{UO}_{2}-\mathrm{CeO}_{2}$ Pellet mit verschieden tiefen Bohrungen in der oberen Stirnfläche parallel zur Zylinderachse. Für diese Messung wurde ein durchbohrter Deckel auf den Probenbehälter gesetzt, damit von oben mit einem Pyrometer die Temperatur in den Bohrungen gemessen werden konnte. Alle Versuche wurden im Hochvakuum durchgeführt. Die Aufheizgeschwindigkeit betrug 
$50^{\circ} \mathrm{C} / \mathrm{min}$. Es musste möglichst vorsichtig aufgeheizt werden, damit die Pellets nicht zersprangen, aber selbst bei langsamem Aufheizen liess sich dies nicht immer vermeiden.

In der Tabelle 1 sind die Versuchsdaten für die einzelnen Proben wiedergegeben. $T_{\max }$ bedeutet dabei die Temperatur, welche an der heissesten Stelle der Probe, also an der oberen Stirnfläche vorhanden war und $T_{\min }$ diejenige, die an der unteren, kälteren Stirnfläche herrschte.

TABeLte 1

\begin{tabular}{|c|c|c|c|c|c|}
\hline $\begin{array}{c}\text { Probe } \\
\text { Nr. }\end{array}$ & $\begin{array}{c}\mathrm{CeO}_{2} \text {-Konz. } \\
(\mathrm{Gew} \%)\end{array}$ & Herstellungsart & $\begin{array}{l}T_{\max } \\
\left({ }^{\circ} \mathrm{K}\right)\end{array}$ & $\begin{array}{l}T_{\min } \\
\left({ }^{\circ} \mathrm{K}\right)\end{array}$ & $\begin{array}{l}\text { Versuchs- } \\
\text { dauer (h) }\end{array}$ \\
\hline 1 & 13.3 & gemeinsame Fällung & 2325 & 1640 & 115 \\
\hline 2 & 13.3 &, & 2375 & 1680 & 185 \\
\hline 3 & 5.6 & , & 2150 & 1700 & 600 \\
\hline 4 & 3.7 & Pulvermischung & 2100 & 1660 & 40 \\
\hline
\end{tabular}

\subsection{KONZENTRATIONSANALYSE}

Nach beendeter Temperaturbehandlung wurde in einem Handschuhkasten das Pellet aus dem Probebehälter entfernt und mit einer Diamantkreissäge axial in zwei Hälften getrennt. Eine Hälfte wurde für die mikroanalytische und mikroskopische Untersuchung in Plexigum eingebettet, geschliffen und poliert. Mit Hilfe eines Ätzpolierverfahrens gelang es, auch von sehr brüchigen Proben für Gefügeuntersuchungen brauchbare Anschliffe zu erhalten ${ }^{10}$ ). Die CeO2-Konzentrationsanalysen wurden mit einer Cameca-Mikrosonde nach dem Scanning-Verfahren ${ }^{11}$ ) durchgeführt. Der Elektronenstrahl wurde dabei auf einer Fläche von $0.7 \times 0.2 \mathrm{~mm}$ Ausdehnung hin- und herbewegt, wobei die längere Seite der Fläche senkrecht zur Pelletachse verlief. Die Fläche wurde verhältnismässig gross gewählt, damit Messwertschwankungen im Mikrobereich ausgeglichen werden. Die Lage der Analysenbereiche wurde so gewählt, dass möglichst kleine strukturbedingten Messfehler auftraten. Der Messfehler der Analyse beträgt maximal $\pm 3 \%$ des gemessenen $\mathrm{CeO}_{2}$-Gehaltes.

\section{Ergebnisse}

\subsection{GEFÜGEÄNDERUNGEN}

In allen Proben gingen während der Temperaturbehandlung auffällige Gefügeänderungen vor sich. Die Körper rekristallisierten und verdichteten sich je nach Versuchsdauer und
Temperatur zum Teil sehr stark. Die Fig. 4-7 zeigen das Gefüge der Proben 1-4 nach der Glühung im Temperaturgradienten. Sie sind so angeordnet, dass im oberen Bildabschnitt jeweils der Probenteil zu sehen ist, welcher der höheren Temperatur ausgesetzt war.

In Fig. 4 ist das Makro-Gefüge der Probe 1 zu sehen. Im oberen, heisseren Bereich ist die Probe stark rekristallisiert. Man kann eine stetige Zunahme der Kristallitgrösse vom unteren bis zum oberen Proberand erkennen. Im oberen Teil des Peltets ist eine breite Reaktionszone mit dem Tantal-Probebehälter vorhanden. Die Risse entstanden beim Abkühlen, das wegen eines Heizerdefekts sehr schnell erfolgte. In der Mitte der Probe ist deutlich eine poröse Zone zu erkennen, die mit dem Minimum der $\mathrm{CeO}_{2}$-Konzentration zusammenfällt. Auf die Ursache dieser porösen Zone wird in der Diskussion (Abschnitt 5.3) eingegangen.

Das Schliffbild der Probe 2 in Fig. 5 zeigt die gleichen Merkmale wie das der Probe 1 in Fig. 4. In der oberen Hälfte hat ebenfalls eine starke Rekristallisation des Gefüges und Reaktion mit der Behälterwand stattgefunden. Das Gefüge im Pellet war teilweise sehr locker, so dass sich beim Trennen mit der Diamantsäge eine grosse Anzahl von Kristalliten herauslöste. In einem breiten Streifen oberhalb der Pelletmitte waren die Materialausbrüche so gross, dass eine tiefe Rinne entstand, die durch das Schleifen nicht mehr beseitigt werden konnte. Die Risse in der 


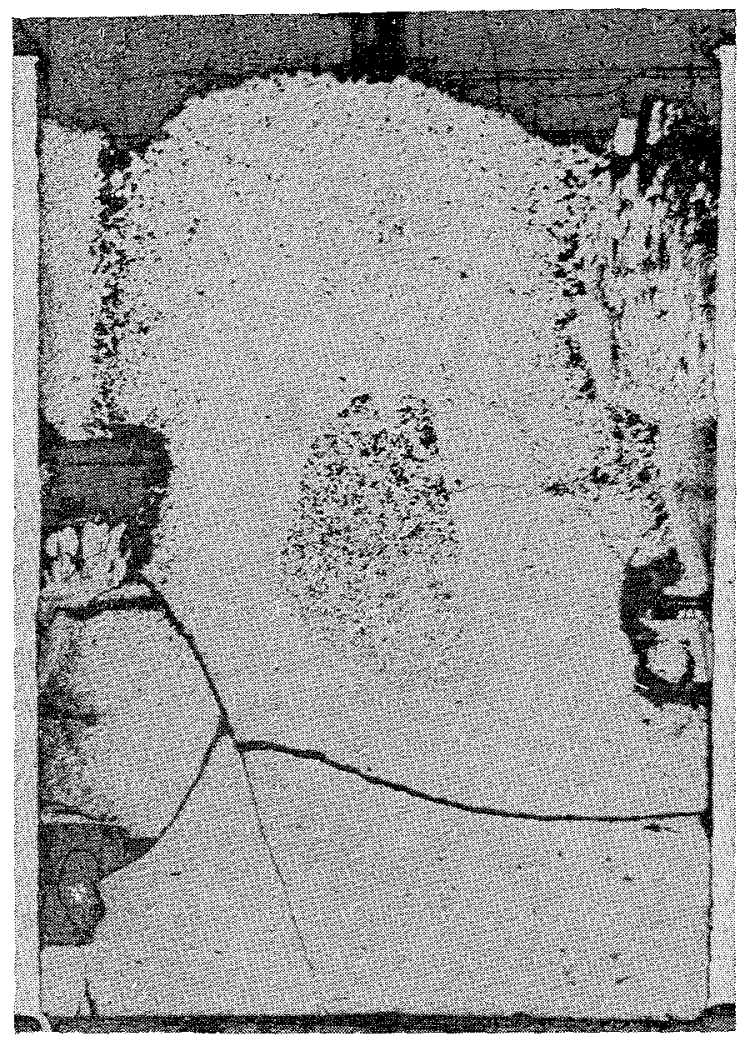

Fig. 4. Probe 1, $T_{\max }=2325^{\circ} \mathrm{K} ; T_{\min }=1640^{\circ} \mathrm{K}$; $t=115 \mathrm{~h} ; \times 12$

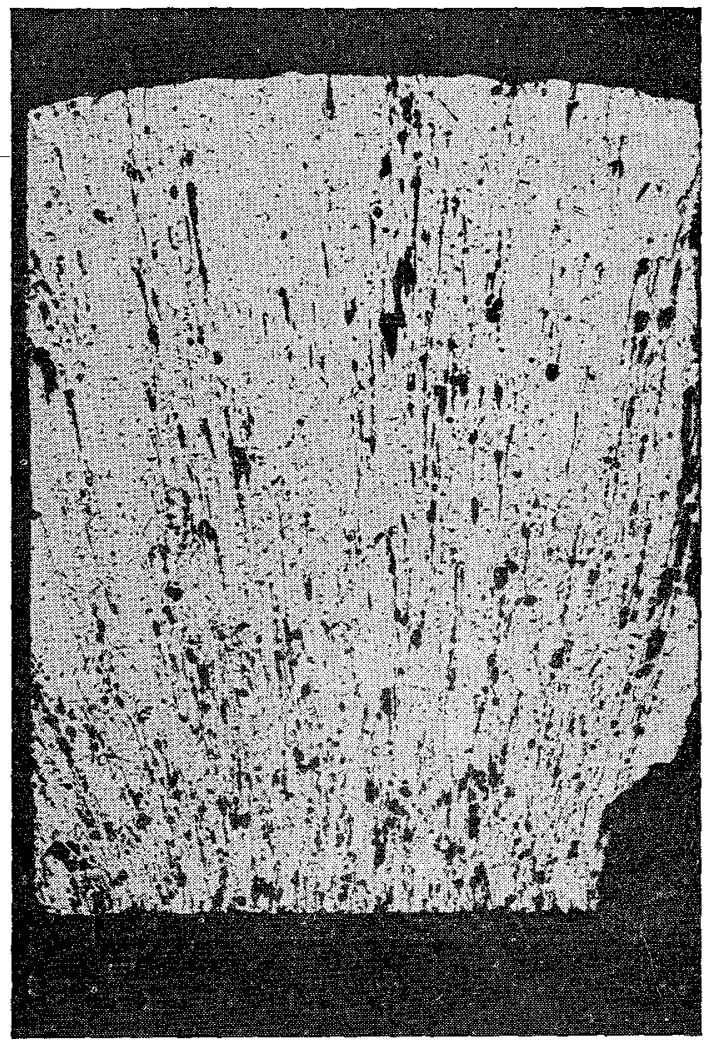

Fig. 6. Probe $3, T_{\max }=2150^{\circ} \mathrm{K} ; T_{\min }=1700^{\circ} \mathrm{K}$; $t=600 \mathrm{~h} ; \times 12$

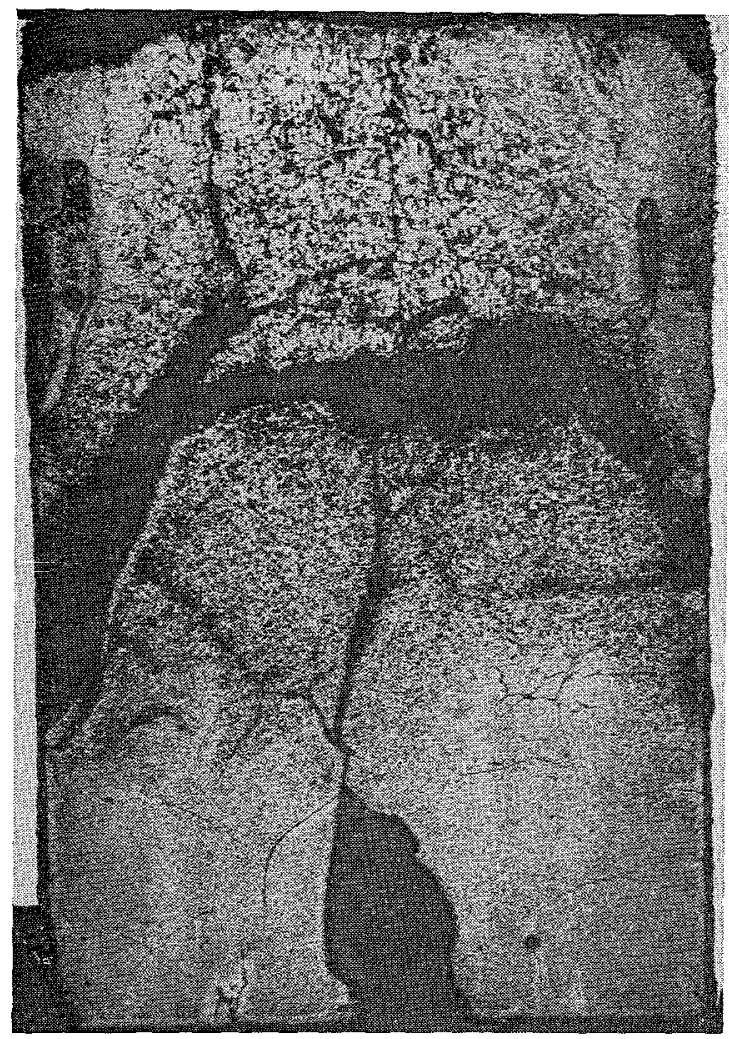

Fig. 5. Probe $2, T_{\max }=2375^{\circ} \mathrm{K} ; T_{\min }=1680^{\circ} \mathrm{K}$; $t=185 \mathrm{~h} ; \times 12$

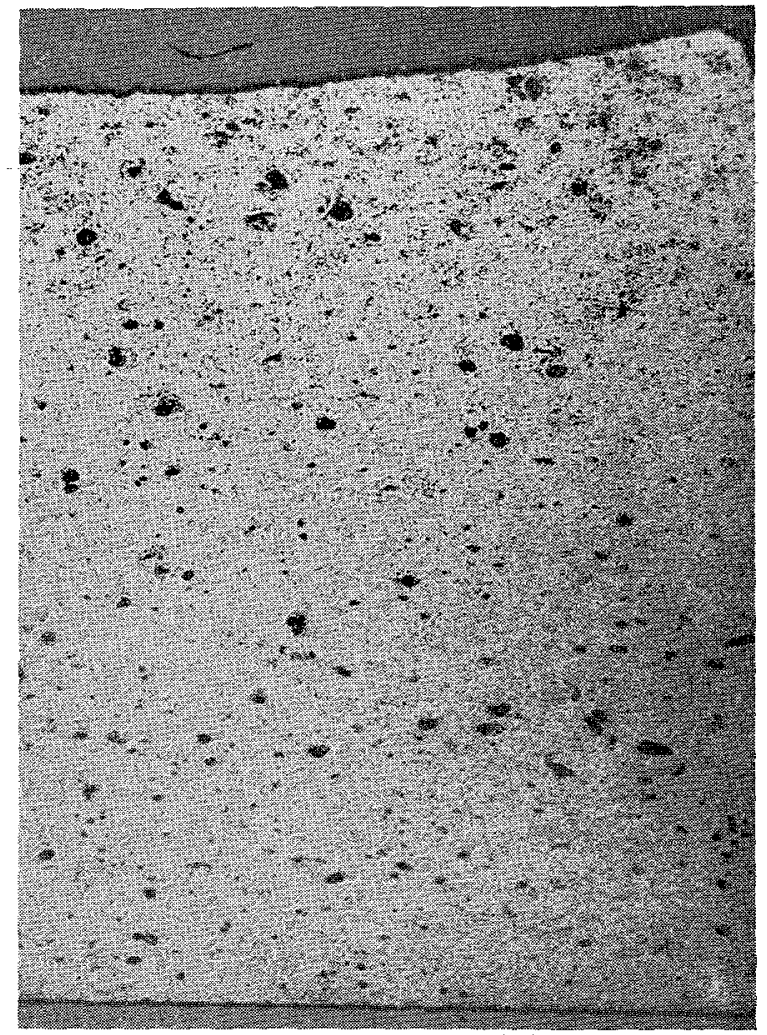

Fig. 7. Probe 4, $T_{\max }=2100^{\circ} \mathrm{K} ; T_{\min }=1660^{\circ} \mathrm{K}$; $t=40 \mathrm{~h} ; \times 12$

Figs. 4-7. Gefügebilder der Proben nach der Glühung im Temperaturgradienten (Proben 1-3, gemeinsam Fällungen; Probe 4 Pulvermischung). 
Probe entstanden ebenfalls beim Abschrecken aufgrund eines Heizerdefekts.

Die Probe 3, Fig. 6, hat ein sehr stark rekristallisiertes Gefüge. Sie besteht vollständig aus Säulenkristallen, die mehrere Millimeter lang sind und die Richtung des Temperaturgradienten haben. Hier hat keine sichtbare Reaktion mit dem Probebehälter stattgefunden. Die Probe konnte ohne Zerstörung des Probebehälters entnommen werden.

Das Gefüge der Probe 4 ist in Fig. 7 wiedergegeben. Das Ausgangsmaterial für die Probe war eine Pulvermischung mit $3.7 \mathrm{Gew} \% \mathrm{CeO}_{2}$. Über die ganze Probe verteilt sind $\mathrm{CeO}_{2^{-}}$ Einschlüsse zu sehen, die sich besonders im oberen Teil in ihrer Umgebung aufgelöst haben. In der Mitte dieser $\mathrm{CeO}_{2}$-Einschlüisse entstehen meistens Hohlräume, was darauf zurückzuführen sein dürfte, dass mehr $\mathrm{CeO}_{2}$ in das $\mathrm{UO}_{2}$ diffundiert als $\mathrm{UO}_{2}$ in das $\mathrm{CeO}_{2}$.

\subsection{KONZENTRATIONSÄNDERUNGEN IM TEMPERATURgRadienteN}

Die gemessenen Konzentrationsänderungen sind grösser als erwartet wurde. Deshalb konnten in gut realisierbaren Glühzeiten sehr deutliche Effekte erzielt werden.

Aus allen Konzentrationsmessungen geht hervor, dass $\mathrm{CeO}_{2}$ in Richtung des positiven Temperaturgradienten wandert. Die Fig. 8-11 geben den Konzentrationsverlauf in den Proben 1-4 nach der Temperaturbehandlung wieder.

In Fig. 8 ist die Konzentrationskurve der Probe 1 dargestellt. Die Probe wurde $115 \mathrm{~h}$ geglüht. Das Diagramm zeigt, dass in dieser Zeit oberhalb $1950^{\circ} \mathrm{K}$ durch Thermodiffusion beträchtliche Konzentrationsänderungen entstehen. Bei $2050^{\circ} \mathrm{K}$ bildet sich durch den $\mathrm{CeO}_{2}$-Transport in Richtung des Temperaturgradienten ein Konzentrationsminimum, da die Diffusion mit tiefer werdenden Temperaturen immer langsamer vonstatten geht. Im oberen Teil der Probe bildet sich ein Konzentrationsmaximum aus, oberhalb dessen die $\mathrm{CeO}_{2}$ Konzentration gegen das obere Ende hin wieder fällt. Dieser Abfall ist wohl darauf zurückzuführen, dass $\mathrm{CeO}_{2}$ vom oberen Ende der Probe an die Umgebung abgegeben wurde.

Der Konzentrationsverlauf in Probe 2 nach $185 \mathrm{~h}$ Glühzeit ist in Fig. $9 \mathrm{zu}$ sehen. Hier liegt ein stark ausgeprägtes Minimum bei $1970^{\circ} \mathrm{K}$. Der nachfolgende Konzentrationsanstieg ist sehr stark. Man kann deshalb annehmen, dass in diesem Bereich annähernd der Konzentrationsgradient des stationären Zustandes erreicht ist.

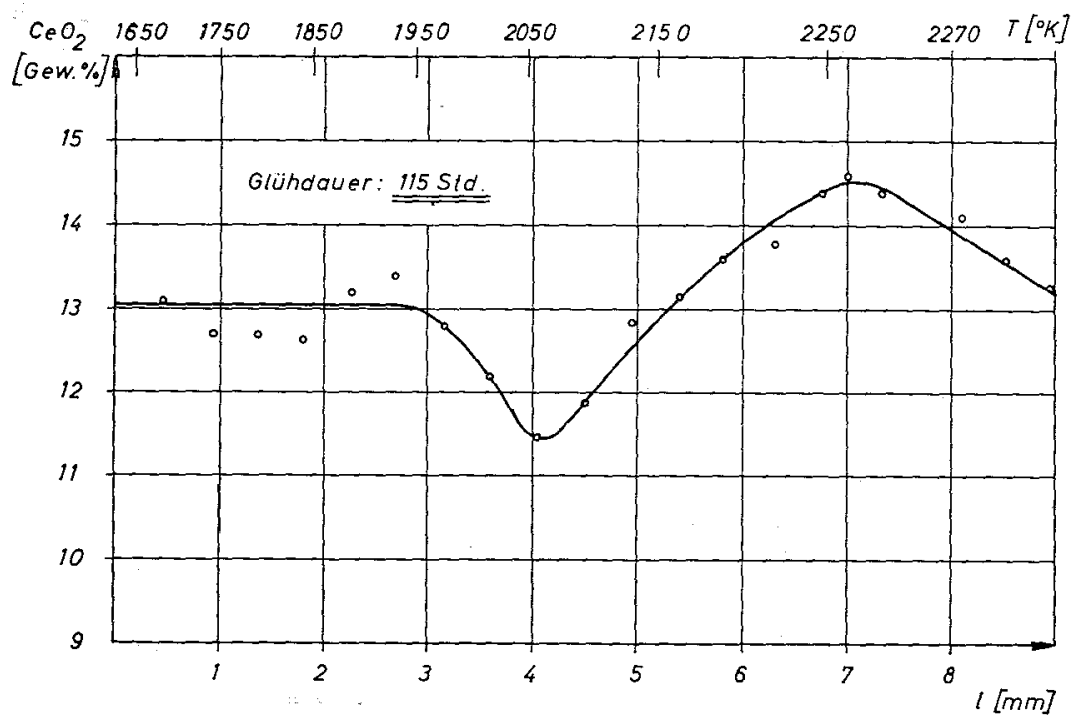

Fig. 8. Konzentrationsverlauf in der Probe $\mathrm{l}$ nach $115 \mathrm{~h}$ Glühung im Temperaturgradienten. $\mathrm{CeO}_{2}$-Ausgangskonzentration: $13.3 \mathrm{Gew} \%$. 


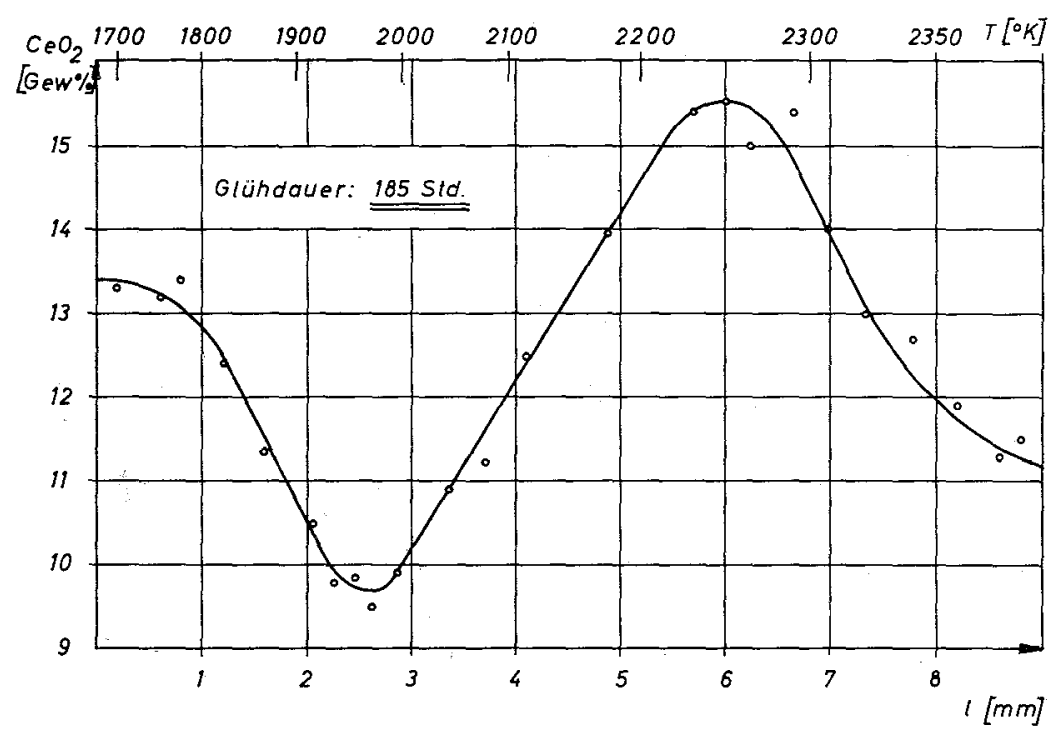

Fig. 9. Konzentrationsverlauf in der Probe 2 nach $185 \mathrm{~h}$ Glühung im Temperaturgradienten. CeO $\mathrm{O}_{2}$-Ausgangskonzentration: 13.3 Gew \%.

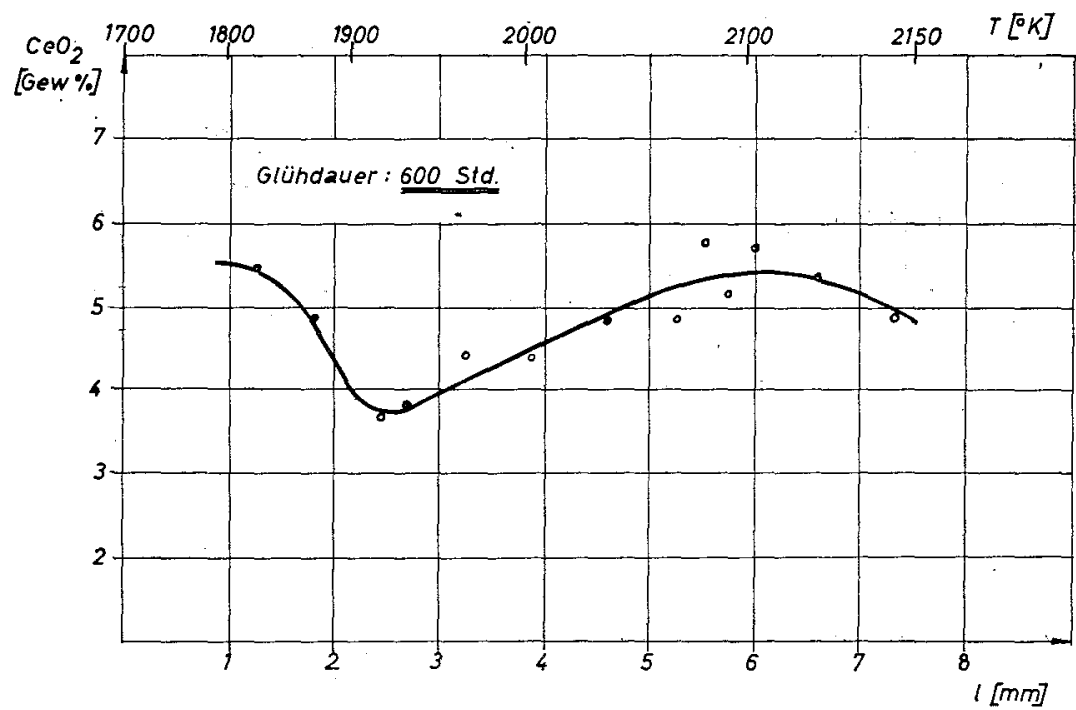

Fig. 10. Konzentrationsverlauf in der Probe 3 nach $600 \mathrm{~h}$ Glühung im Temperaturgradienten. CeO ${ }_{2}$-Ausgangskonzentration: 5.6 Gew \%.

In der Probe 3 ist, bedingt durch die lange Glühzeit von 600 Stunden, die $\mathrm{CeO}_{2}$-Konzentration im Maximum unter die des Ausgangszustandes (5.6 Gew \% $\mathrm{CeO}_{2}$ ) gesunken (Fig. 10). Die Steigung ist flacher, da der Temperaturgradient hier nur halb so gross wie bei den Proben 1 und 2 ist.

Die Probe 4 hat nur eine Ausgangskonzen- tration von 3.7 Gew \% $\mathrm{CeO}_{2}$. Hinzu kommt, dass ein Teil des $\mathrm{CeO}_{2}$ aufgrund der groben Pulvermischungen im Ausgangszustand nicht in Lösung war und auch nach der Temperaturbehandlung noch $\mathrm{CeO}_{2}$-reiche Inseln existierten. Es konnte also nur ein Teil des $\mathrm{CeO}_{2}$ an der Thermodiffusion teilnehmen. Das bedeutet, dass zu geringe Konzentrationsänderungen ge- 


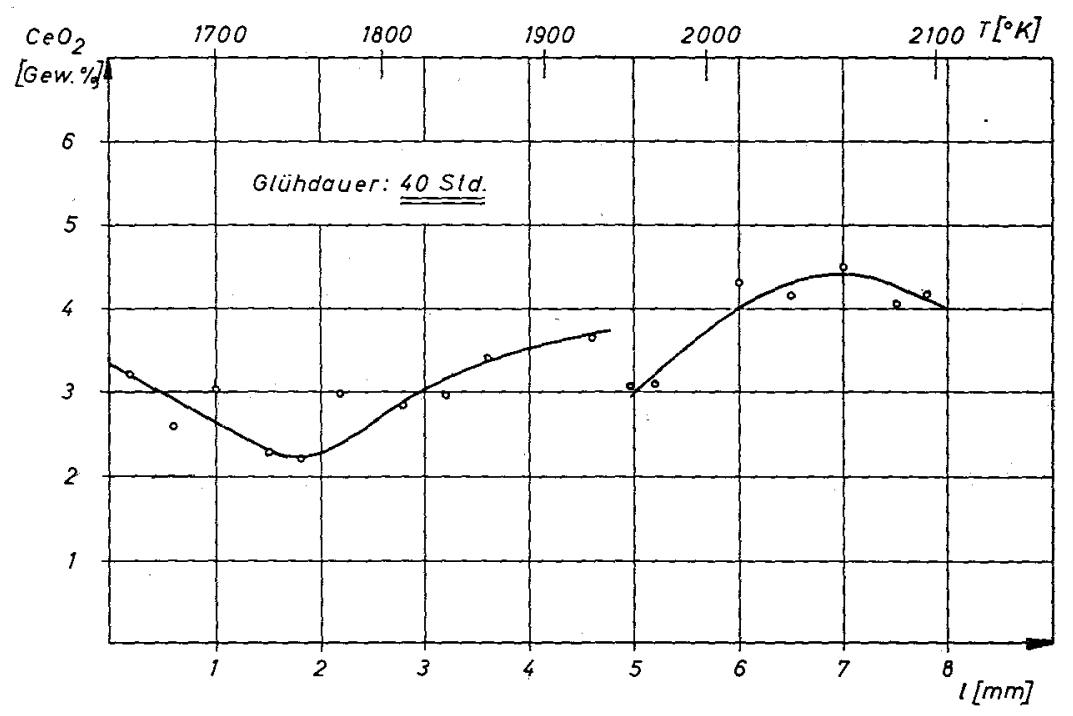

Fig. 11. Konzentrationsverlauf in der Probe 4 nach $40 \mathrm{~h}$ Glühung im Temperaturgradienten. $\mathrm{CeO}_{2}$-Ausgangskonzentration: 3.7 Gew $\%$.

messen wurden. Das Konzentrationsminimum (Fig. 11) liegt bei der relativ niedrigen Temperatur von $1750^{\circ} \mathrm{K}$. Das ist wahrscheinlich darauf zurückzuführen, dass der Thermodiffusionseffekt mit abnehmender Konzentration stärker wird.

Die Konzentrationskurve in Fig. 11 zeigt noch eine Besonderheit. Etwas oberhalb der Probenmitte zeigt der Konzentrationsverlauf einen Sprung. Der Sprung in der Konzentrationskurve wurde wahrscheinlich durch einen Riss in der Probe hervorgerufen. Dieser Riss kann beim Sintern oder Aufheizen des Pellets entstanden sein. Er heilte während der Temperaturbehandlung offenbar $\mathrm{zu}$, da er in den Schliffbildern nicht zu sehen ist. Während des Versuchs muss er über eine grosse Zeitspanne hinweg als Diffusionsbarriere gewirkt und so zur Entstehung eines zweiten Minimums beigetragen haben.

4.3. Vorzugstichtong DER DIFFuston BeI DFR AUFLÖSUNG DER $\mathrm{CeO}_{2}$-INSELN

Die aus Pulvermischungen hergestellten Proben enthalten im Ausgangszustand über das ganze Volumen gleichmässig verteilt Inseln, die aus $\mathrm{CeO}_{2}$ bestehen (Fig. 3).

Anhand einer Probe, die 8 Stunden in einem
Temperaturgradienten von $2350-1900^{\circ} \mathrm{K}$ geglüht wurde, wurde die Aufösung einiger Inseln in einem mittleren und oberen Temperaturbereich näher untersucht. Die Bilder sind wiederum so angeordnet, dass der Temperaturgradient von der unteren zur oberen Bildkante weist.

Das Gefüge der Probe im mittleren Temperaturbereich, der von $2160^{\circ} \mathrm{K}$ bis $2190^{\circ} \mathrm{K}$ reicht, ist in Fig. 12 dargestellt. Das $\mathrm{CeO}_{2}$ ist unter Bildung einer ringförmigen Diffusionszone in seine Umgebung hineindiffundiert. Der Teil der Diffusionszone, der zur oberen Bildkante, also zur höheren Temperatur gekehrt ist, hat sich wesentlich breiter ausgebildet. Noch stärker ist dieser Effekt im oberen Temperaturbereich $\left(2300^{\circ} \mathrm{K}\right.$ bis $2330^{\circ} \mathrm{K}$ ) ausgeprägt (Fig. 13).

Bei den mikroanalytischen Untersuchungen der Ringzonen ergab sich, dass in Richtung des positiven Temperaturgradienten ein flacherer Abfall der $\mathrm{CeO}_{2}$-Konzentration und damit ein stärkerer Diffusionseffekt als in Richtung niedrigerer Temperaturen vorhanden ist.

Der Effekt der richtungsabhängigen Diffusion bei der Auflösung der $\mathrm{CeO}_{2}$-Inseln ist darauf zurückzuführen, dass in Richtung des positiven Temperaturgradienten die Diffusion durch den Thermodiffusionseffekt verstärkt und in der entgegengesetzten Richtung abgeschwächt wird. 


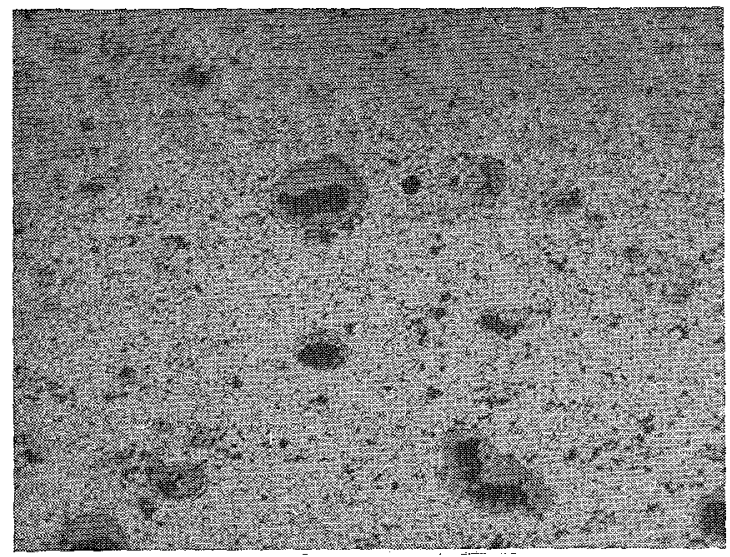

Fig. 12. Mittlerer Temperaturbereich $2160-2190^{\circ} \mathrm{K}$; $\times 100$

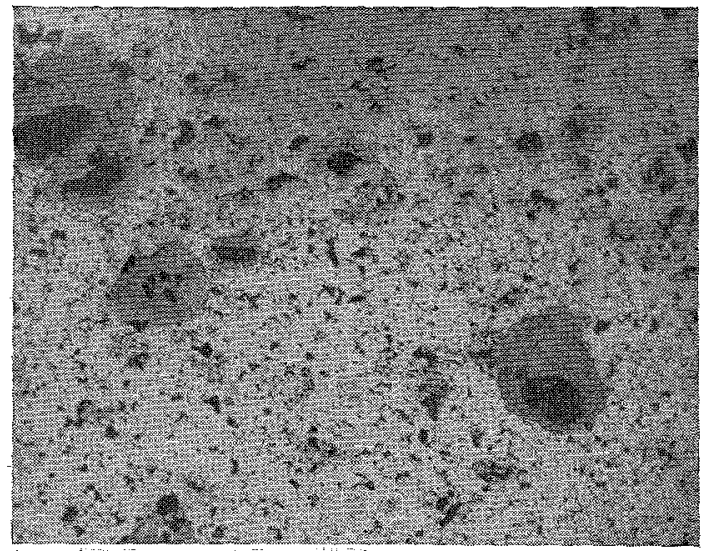

Fig. 13. Oberer Temperaturbereich $2300-2330^{\circ} \mathrm{K}$; $\times 100$

Figs. 12-13. Auflösung der $\mathrm{CeO}_{2}$-Inseln in verschiedenen Temperaturbereichen nach 8 Stunden Glühung im Temperaturgradienten.

\section{Diskussion}

\subsection{Konzentrationsvertadf als Folge DeR Thermodiffuston}

Die hauptsächliche Auswirkung der Glühung im Temperaturgradienten ist die Wanderung von $\mathrm{CeO}_{2}$ zur höheren Temperatur aufgrund des Thermodiffusionseffekts. Dieser Vorgang verläuft bei der tieferen Temperatur langsam und bei der höheren Temperatur schneller. Dadurch wird im unteren Teil der Probe $\mathrm{CeO}_{2}$ abgetragen und im oberen Teil angehäuft. Dieser Prozess geht solange, bis sich ein Konzentrationsgradient einstellt, bei dem sich Thermodiffusion und Diffusion die Waage halten und somit ein stationärer Zustand erreicht ist.

Der zeitliche Verlauf vollzieht sich so, dass zunächst ein flaches Minimum bei verhältnismässig hoher Temperatur entsteht und am oberen Ende der Probe die Konzentration ansteigt. Im Laufe der Zeit wird das Minimum immer tiefer und das Maximum höher. Gleichzeitig verschiebt sich das Minimum zu tieferen Temperaturen.

\section{2. $\mathrm{CeO}_{2}$-Verlust ain oberen Probenende}

In allen untersuchten Proben nimmt die $\mathrm{CeO}_{2}$-Konzentration gegen das obere Ende hin wieder ab. Dieser Abfall ist mit dem beschrie- benen Thermodiffusionsmechanismus nicht zu erklären sondern nur durch eine $\mathrm{CeO}_{2}$-Abgabe nach aussen. Es ist möglich, dass das $\mathrm{CeO}_{2}$ in stärkerem Masse mit der Behälterwand reagiert als $\mathrm{UO}_{2}$ und dadurch eine Abreicherung an $\mathrm{CeO}_{2}$ stattfindet. Dieser Prozess läuft in verstärktem Masse ab, wenn ein direkter Kontakt vorhanden ist (Fig. 4 und 5), der durch allmähliches Zudampfen des Spaltes zwischen Probe und Probebehälter entsteht.

In dem Bereich der Konzentrationsabnahme spielt die Thermodiffusion keine grosse Rolle, da der Temperaturverlauf zum oberen Probenende hin sehr flach wird. Wie im Abschnitt 6 erläutert wird, kann dieser Bereich bei der Auswertung unberücksichtigt bleiben.

\subsection{Porosität im Probeninnern - WANDERUNGen Der LEerstellen}

In mehreren Proben, besonders deutlich in Fig. 4, wurde eine poröse Zone gefunden. Diese Zone fällt ungefähr mit dem Konzentrationsminimum zusammen. Sie liegt also in dem Bereich, wo $\mathrm{CeO}_{2}$ durch. Thermodiffusion abgewandert ist. Da die Diffusion im allgemeinen über Leerstellen erfolgt, muss eine LeerstellenWanderung entgegengesetzt zur $\mathrm{CeO}_{2}$-Wanderung auftreten. Diese Leerstellen häufen sich dann an den Stellen, aus denen das $\mathrm{CeO}_{2}$ 
abgewandert ist and schliessen sich im Laufe der Zeit zu Poren zusammen.

Ausser der Wanderung von $\mathrm{CeO}_{2}$ kann auch eine gewisse $\mathrm{Abwanderung}$ von $\mathrm{UO}_{2}$ erfolgt sein und Leerstellen erzeugt haben. Wenn die durch Leerstellen entstandene Porosität bekannt wäre, könnten auch Aussagen über die $\mathrm{UO}_{2}$-Thermodiffusion gemacht werden. Eine quantitative Bestimmung der Porosität war bei der vorliegenden Arbeit noch nicht möglich.

Bei sehr langen Glühzeiten kann es zur Ruekristallisation kommen, wobei die Poren wieder verschwinden bzw. an das heisse Ende der Probe wandern. Diese Erscheinung kann an Probe 3 (Fig. 6) beobachtet werden.

\subsection{Wanderung DER SpautprodukTE IN bestrahlten Proben}

Bei bestrahlten Proben wird in mehreren Arbeiten von Bates und Mitarbeitern etwa folgender Verlauf der Ce-Konzentration festgestellt:

Aussen ist eine Zone, in der die Konzentration im wesentlichen unverändert bleibt. Dann kommt ein plötzlicher Abfall und ein erneuter Anstieg bis zu einem Maximum und ein allmählicher Abfall bis zum Brennelementzentrum.

Qualitativ stimmt somit der Verlauf in einem Brennelement mit unseren Ergebnissen überein. Fine quantitative Übereinstimmung ist schwierig nachzuweisen, da der Temperaturverlauf im Brennelement nicht genügend genau bekannt ist.

Der erste plötzliche Abfall der Konzentration in den bestrahlten Proben erfolgt an einer Stelle, an der im Schliffbild deutlich Risse zu erkennen sind, die quer zum Temperaturgradienten verlaufen. Die Risse wirken offenbar als Diffusionsbarrieren. Ahnliche Beobachtungen konnten auch bei unseren Versuchen gemacht werden, wie Fig. 7 (Probe 4) zeigt. An der Stelle des Risses macht die Konzentrationskurve einen Sprung, da der Transport des $\mathrm{CeO}_{2}$ an dem Riss unterbrochen ist.

An der heissesten Stelle in der Mitte des Brennelements wird ein flacher Abfall der $\mathrm{CeO}_{2}$-Konzentration gefunden. Dies kann die
Folge einer bevorzugten $\mathrm{CeO}_{2}$-Verdampfung sein, wobei das $\mathrm{CeO}_{2}$ über den zentralen Kanal in axialer Richtung abtransportiert wïrde.

Die anderen Spaltprodukte verhalten sich ähnlich wie das Ce. So zeigt z.B. Zr, Nb, Ru und $O$ s einen steilen Konzentrationsabfall in der Risszone wie das Ce. Gegen die hohe Temperatur wird ein Konzentrationsanstieg beobachtet, der in einigen Fällen zu einem Maximum in der Mitte führt. In anderen Fällen wird wie beim $\mathrm{Ce}$ in der Mitte eine flache Einbeulung, also Erniedrigung der Konzentration, beobachtet.

\section{Berechnung des Diffusions-, des Thermo- diffusions- und des Soret-Koeffizienten}

Nach de Groot und Mazur ${ }^{2}$ ) beträgt der Strom ${ }_{s} J_{1}$ einer Komponente 1 in einem Temperaturgradienten $\partial T / \partial x$

$$
\begin{aligned}
& \mathrm{s} J_{1}=-D\left(\mathrm{\partial} c_{1} / \partial x\right)+D^{\prime}\left(c_{1} c_{2} / \varrho\right)(\mathrm{d} T / \mathrm{\partial} x), \quad(\mathrm{I}) \\
& \varrho=\left(m_{1}+m_{2}\right) / V=c_{1}+c_{2}, \\
& m_{1}= \text { Masse der Komponente } 1[\mathrm{~g}], \\
& m_{2}=,, \quad 2[\mathrm{~g}], \\
& V= \text { Volumen }\left[\mathrm{cm}^{3}\right], \\
& c_{1}= m_{1} / V=\text { Massenkonzentration der Komp. } \\
& 1\left[\mathrm{~g} / \mathrm{cm}^{3}\right], \\
& c_{2}= m_{2} / V=\text { Massenkonzentration der Komp. } \\
& 2\left[\mathrm{~g} / \mathrm{cm}^{3}\right], \\
& D= \text { Diffusionskoeffizient }\left[\mathrm{cm}^{2} / \mathrm{sec}\right], \\
& D^{\prime}= \text { Thermodiffusionskoeffizient } \\
& {\left[\mathrm{cm}^{2} / \mathrm{sec} \cdot{ }^{\circ} \mathrm{K}\right], } \\
& T= \text { Temperatur }\left[{ }^{\circ} \mathrm{K}\right] .
\end{aligned}
$$

Wie Gleichung (1) zeigt, setzt sich der Strom in einem Temperaturfeld, wenn keine elektrischen Kräfte auftreten, aus 2 Anteilen zusammen. Der erste Anteil rührt von der normalen Diffusion her und ist dem Konzentrationsgradienten $\partial c / \partial x$ proportional. Der andere rührt von dex Thermodiffusion her und ist dem Temperaturgefälle $\partial T / \partial x$ proportional. Elektrische Potentialdifferenzen, die durch Unterschiede in den Ionenkonzentrationen entstehen können, werden bei unserer Betrachtung vernachlässigt, weil die Probekörper in unseren Versuchen all- 
seitig guten Kontakt mit den metallenen Probebehältern hatten.

Gleichung (1) gilt für das Schwerpunktsystem. Dieses ist für unseren Fall unzweckmässig. Wir rechnen deshalb auf ein ortsfestes Gitterbezugssystem um [sh. Haase 12)]. Die Umrechnung wird im Anhang ausgeführt. Wir erhalten

$$
{ }_{\mathrm{G}} J_{1}=-D\left\{1+\left(c_{1} / c_{2}\right)\right\} \partial c_{1} / \partial x+D^{\prime} c_{1} \partial T / \partial x .
$$

In Gleichung (1) und (2) wird nur der Strom der Komponente 1 in Betracht gezogen. Die Konzentrationsänderungen, die durch die Wanderung der Komponente 2 hervorgerufen werden, wurden vernachlässigt. Das bedeutet, dass die Massenkonzentration $c_{2}$ konstant bleibt. Auch die Wanderung von Gitterfehlstellen, z.B. Leerstellen, über die die Diffusion im wesentlichen erfolgt ${ }^{13}$ ), lassen wir ausser Betracht. Die Vernachlässigung der $\mathrm{UO}_{2}$-Wanderung erscheint uns aufgrund der Grösse der hier in relativ kurzen Zeiten erzielten Konzentrationsänderungen gerechtfertigt. Die bekannten Selbstdiffusionskoeffizienten von $\mathrm{U}$ in $\mathrm{UO}_{2}{ }^{16-19}$ ) sind viel zu klein für die Erzeugung wesentlicher U-Konzentrationsverschiebungen in solchen Zeiträumen.

Eine umfassendere Berechnung mit Berücksichtigung des $\mathrm{UO}_{2^{-}}$und Leerstellenstroms kann erst dann vorgenommen werden, wenn Messungen über diese Grössen vorliegen. Bis jetzt sind jedoch solche Messungen noch nicht durchgeführt worden.

Zur Berechnung des Diffusions- $D$ und Thermodiffusionskoeffizienten $D^{\prime}$ ist es zweckmässig, deren Temperaturabhängigkeit explizit einzusetzen. Sowrohl $D$ als auch $D^{\prime}$ enthalten einen Faktor der Form $\exp (-Q / R T)$. Wir setzen daher

$$
\begin{aligned}
& D=D_{0} \exp (-Q / R T), \\
& D^{\prime}=D_{0}{ }^{\prime} \exp (-Q / R T) .
\end{aligned}
$$

Der Thermodiffusionskoeffizient $D^{\prime}$ enthält ausserdem einen Faktor $1 / T$ [de Groot ${ }^{2}$ ) und Haase $\left.{ }^{12}\right)$ ]. Er lässt sich damit schreiben:

$$
D^{\prime}=D_{0}^{*}(1 / T) \exp \left(-Q / R T^{\prime}\right) .
$$

Aus (2), (3) und (5) ergibt sich

$$
\begin{gathered}
{ }_{\mathrm{G}} J_{1}=\{\exp (-Q / R T)\}\left\{-D_{0}\left(1+c_{1} / c_{2}\right) \partial c_{1} / \partial x+\right. \\
\left.+D_{0}^{*} c_{1} 1 / T \partial T / \partial x\right\},
\end{gathered}
$$

wobei die Aktivierungsenergie $Q$ und die Koeffizienten $D_{0}$ und $D_{0}{ }^{*}$ in dem betrachteten Temperaturbereich als konstant angesehen werden.

Neben dieser Gleichung muss noch die Erhaltung der Masse verlangt werden. Dies lässt sich in folgender Form wiedergeben:

$$
\partial J_{1} / \partial x=-\partial c_{1} / \partial t .
$$

Aus den Gleichungen (6) und (7) kann für vorgegebene Werte $D_{0}, D_{0}{ }^{*}$ und $Q$ der zeitliche und räumliche Konzentrationsverlauf $c_{1}$ errechnet werden. Der Temperaturverlauf, der zeitlich konstant ist und die Ausgangskonzentration werden als bekannt vorausgesetzt. Die Rechnung wurde mit der Rechenmaschine IBM 7070 durchgeführt; dabei wurden die Werte von $D_{0}, D_{0}$ * und $Q$ solange variiert, bis die errechnete Konzentrationskurve im charakteristischen Kurventeil eine befriedigende Übereinstimmung mit der gemessenen Kurve ergab. Bestimmend für die Grösse $Q$ ist der Kurvenverlauf links von Minimum und für die Grössen $D_{0}$ und $D_{0}{ }^{*}$ die Lage und Tiefe des Minimums sowie die Steilheit des Konzentrationsanstiegs in Abhängigkeit von der Glühzeit. Im Anhang wird die Durchführung der Rechnung beschrieben.

In der Rechnung wurde die im Absatz 5.2 beschriebene Konzentrationsabnahme am oberen Probenende nicht berücksichtigt. Die Probe wurde als abgeschlossenes System behandelt und der Diffusionsstrom am Rand gleich Null gesetzt. In Fig. 14 sind für verschiedene Zeiten die Konzentrationskurven wiedergegeben, die mit einer Anfangskonzentration von 13.3 Gew $\% \mathrm{CeO}_{2}$ und dem Temperaturgradienten der Probe 2 (Fig. 9) errechnet wurden. Aus der Kurvenschar ist die mit fortschreitender Zeit erfolgende Verschiebung des Minimums nach tieferen Temperaturen ersichtlich.

Mit den folgenden Werten konnte die in Fig. 9 wiedergegebene experimentell erhaltene 


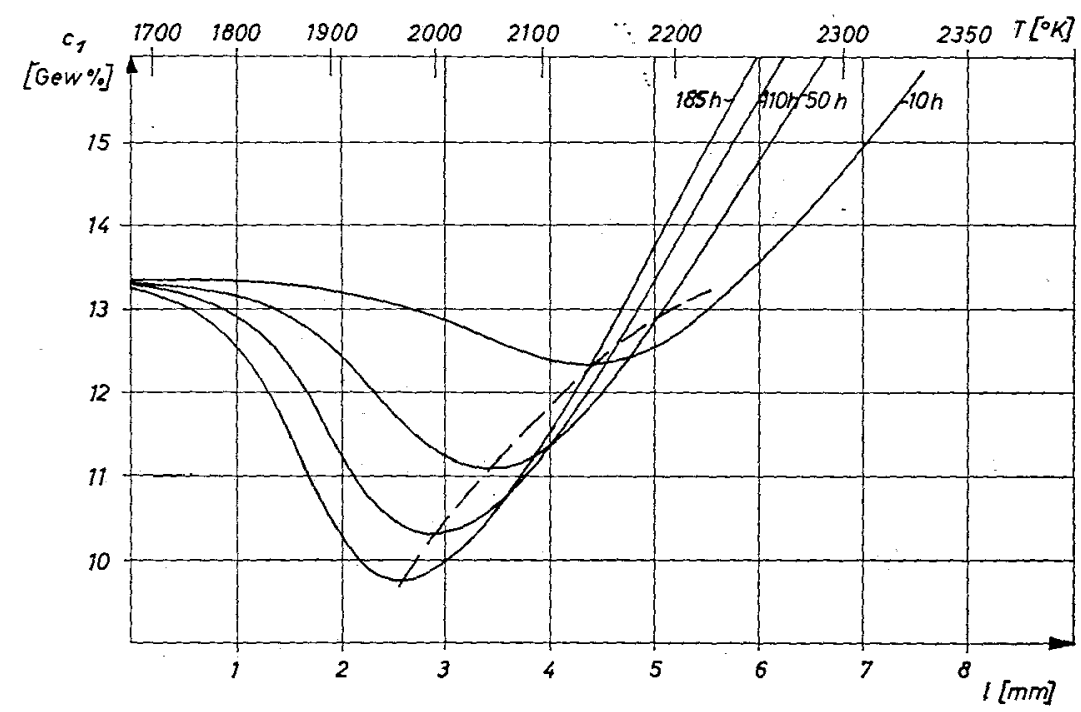

Fig. 14. Errechneter Konzentrationsverlauf für verschiedene Glühzeiten unter den Versuchsbedingungen der Probe 2. Ausgangskonzentration: 13.3 Gew \%.

Konzentrationsku ve am besten nachgebildet werden.

$$
\begin{aligned}
& D=5.4 \times 10^{-8} \mathrm{~cm}^{2} / \mathrm{sec}, \\
& D^{\prime}=1.6 \times 10^{-10} \mathrm{~cm}^{2} / \mathrm{sec} \cdot{ }^{\circ} \mathrm{K}, \\
& Q=110 \mathrm{kcal} / \mathrm{mol} .
\end{aligned}
$$

Diese Werte beziehen sich auf $2050^{\circ} \mathrm{K}$ und $13.3 \mathrm{Gew} \% \mathrm{CeO}_{2}$. Bei Gültigkeit der für die Rechnung gemachten Annahmen ergibt sich für die errechneten Grössen eine Genauigkeit von $\pm 10 \%$. Von Oi und Takagi ${ }^{14}$ ) wurde die Diffusionskonstante und die Aktivierungsenergie ebenfalls gemessen. Thre Messwerte weichen stark von unseren ab. Eine Erklärung hierfür wurde noch nicht gefunden.

Aus dem Thermodiffusionskoeffizienten $D^{\prime}$ und dem Diffusionskoeffizienten $D$ lässt sich der Soret-Koeffizient bestimmen:

$$
S_{T}=D^{\prime} \mid D \text {. }
$$

In unserem Falle wird der Soret-Koeffizient $S_{2050}=3 \times 10^{-3}{ }^{\circ} \mathrm{K}^{-1}$.

Der Soret-Koeffizient lässt sich auch direkt aus der Steilheit einer Konzentrationskurve bestimmen, wenn der stationäre Zustand erreicht ist. Es gilt dann ${ }^{15}$ )

$$
S_{T}=\left\{\left(c_{1}+c_{2}\right) / c_{1} c_{2}\right\} \partial c_{1} / \partial T \text {. }
$$

Unter der Annahme, dass der Konzentrationsanstieg bei $2050^{\circ} \mathrm{K}$ in Probe 2 (Fig. 9) nach 185 h Glühżeit annähernd dem des stationären Zustandes entspricht, erhält man nach (9) $S_{2050}=2.2 \times 10^{-3}{ }^{\circ} \mathrm{K}^{-1}$. Dieser Wert ist kleiner als der aus den Diffusionskoeffizienten errechnete, weil der stationäre Zustand noch nicht erreicht war. Aus dem Konzentrationsverlauf (Fig. 10), der sich nach $600 \mathrm{~h}$ Glühzeit in Probe 3 einstellt, ergibt sich nach (9) ein Soret-koeffizient $S_{2050}=2.8 \times 10^{-3}{ }^{\circ} \mathrm{K}^{-1}$. Dieser Wert kommt dem aus den Diffusionskoeffizienten errechneten erwartungsgemäss näher.

\section{Anhang}

7.1. BereChNutg Des Difhustonsstromes tM GITTERBEZTGSSYSTEM

Der Strom der Komponente 1 wird wie folgt definiert ${ }^{12}$ ):

Im Schwerpunktsystem

$$
{ }_{\mathrm{s}} J_{1}=c_{1}\left(v_{1}-v\right)
$$

und im ortsfesten Gitterbezugssystem

$$
{ }_{\mathrm{G}} J_{1}=c_{1} v_{1},
$$

$v_{1}, v_{2}=$ Diffusionsgeschwindigkeiten der Komponenten 1 und 2,

$v \quad=$ Geschwindigkeit des Schwerpunktes. 
Die Schwerpunktgeschwindigkeit erhält man aus:

$$
v=\left(c_{1} v_{1}+c_{2} v_{2}\right) /\left(c_{1}+c_{2}\right) .
$$

Unter der Annahme, dass die Komponente 2 ein ortsfestes Gitter bildet, wird die Geschwindigkeit $v_{2}$ gleich Null gesetzt. Damit erhält man den Strom ${ }_{\mathrm{G}} J_{1}$ der Komponente 1 im Gitterbezugssystem.

$$
{ }_{\mathrm{G}} J_{1}={ }_{\mathrm{S}} J_{1}+c_{1} v={ }_{\mathrm{S}} J_{1}+\left\{c_{1} /\left(c_{1}+c_{2}\right)\right\} \mathrm{G}_{\mathrm{G}} J_{1}
$$

Durch Einsetzen von Gleichung (1) in (12) folgt:

$$
{ }_{\mathrm{G}} J_{1}=-D\left\{1+\left(c_{1} / c_{2}\right)\right\} \partial c_{1} / \mathrm{\partial} x+D^{\prime} c_{1} \partial T / \mathrm{\partial} x
$$

\subsection{BERECHNUNG DES ZEITLICHEN UND RÄUM- LICHEN KONZENTRATIONSVERLAUTS $c_{1}(x, t)$}

Es wurde ein Rechenprogramm für die IBM 7070 erstellt, das den Konzentrationsverlauf $c_{1}(x, t)$ in vorgegebenen Zeitschritten $\mathrm{d} t$ sukzessiv zu berechnen gestattet.

Aus einer zum Zeitpunkt $t$ vorgegebenen Konzentrationsverteilung $c_{1}(x, t)$ erhält man die Verteilung zum Zeitpunkt $t+\mathrm{d} t$ durch:

$$
c_{1}(x, t+\mathrm{d} t)=c_{1}(x, t)+\left\{\partial c_{1}(x, t) / \partial t\right\} \mathrm{d} t .
$$

Mit Hilfe von Gleichung (7) kann dafür geschrieben werden:

$$
c_{1}(x, t+\mathrm{d} t)=c_{1}(x, t)-\left\{\partial J_{1}(x, t) / \partial x\right\} \mathrm{d} t .
$$

Beginnend mit der Anfangskonzentration wird aus der Konzentrationsverteilung $c_{1}(x, t)$ nach Gleichung (6) der Strom ${ }_{\mathrm{G}} J_{1}(x, t)$ und daraus der Gradient des Stromes $\partial_{G} J_{1}(x, t) / \partial x$ zum Zeitpunkt $t$ in Abhängigkeit vom Ort numerisch berechnet. Nach Gleichung (13) ergibt sich damit die Konzentrationsverteilung $c_{1}(x, t+\mathrm{d} t)$ für den späteren Zeitpunkt $t+\mathrm{d} t$. Durch wechselweises Anwenden von Gleichung (6) und (13) lässt sich der Konzentrationsverlauf für jeden beliebigen Zeitpunkt schrittweise ermitteln. Die Gesamtrechenzeit wird wesentlich durch die Anzahl der erforderlichen Zeitschritte bestimmt. Für die Zeitschrittlänge gibt es eine kritische obere Schranke, die nicht überschritten werden darf. Ihre Grösse hängt von der Feinheit der Ortskoordinatenaufteilung zur numerischen Differenziation und von der Grösse des Faktors $D_{0} \exp (-Q / R T)$ ab. Oberhalb dieser Schranke treten Instabilitäten im numerischen Rechenablauf auf.

Durch Variation der Werte $D_{0}, D_{0}{ }^{*}$ und $Q$ werden die errechneten Konzentrationskurven an die Messkurven angepasst. Die richtige Wiedergabe der Lage und der Tiefe des Minimums in der Messkurve ist nur durch eine eindeutige Wertekombination von $D_{0}, D_{0}{ }^{*}$ und $Q$ möglich. Jede Änderung einer der Werte um etwa $10 \%$ wirkt sich in einer deutlichen Änderung im Verlauf der Konzentrationskurve aus, so dass $10 \%$ Abweichung als obere Fehlergrenze der Rechnung angesehen werden kann.

Die Anregung zu dieser Arbeit wurde von Herrn Prof. K. Wirtz gegeben, der ihrem Fortgang stets sein förderndes Interesse widmete. Wir danken den Herren Dr. G. Giacchetti, Dr. E. Patrassi und Dr. R. Theisen, alle am Institut für Material- und Festkörperforschung des Kernforschungszentrums, für die Durchführung der mikroanalytischen Untersuchungen und Herrn A. Hünlich für die Herstellung der metallographischen Schliffe.

\section{Literatur}

1) K. Wirtz, Z. Physik 124 (1948) 482

2) S. R. de Groot und P. Mazur, Non-equilibrium thermodynamics (North-Holland Publishing Company, Amsterdam, 1963)

3) T. S. Lundy und F. R. Winslow, Atomic transport problems of interest in nuclear systems (IAEA Symposium, Vienna, July 1965)

4) H. Rickert und C. Wagner, Ber. d. Bunsenges. 67, 7 (1963) 621

5) J. L. Bates, J. A. Christensen und W. E. Roake, Nucleonics 20, 3 (1962) 88

6) J. L. Bates, J. A. Christensen und W. E. Roake, Fission fragment migration in $\mathrm{UO}_{2}, \mathrm{HW} 76300$

7) J. L. Bates, Ionic diffusion in high temperature uranium dioxide (TAEA Symposium, Vienna, Juli 1965)

8) N. Oi und J. Takagi, J. Nucl. Sci. 'Techn. 2, 4 (1965) 127

9) N. Oi, T. Ohno und J. Naito, J. Nucl. Sci. Techn. 1, 8 (1964) 284

10) A. Hünlich, Methoden zur Schliffherstellung von $\mathrm{UO}_{2}$ und $\mathrm{UO}_{2}-\mathrm{CeO}_{2}$, Prakt. Metallographie (1966) 
11) A. A. Bauer, H. Beisswenger, G. Giacchetti, E. Patrassi, G. Schumacher und R. Theisen, Microanalytical studies of micro- and macrosegregation in oxide fuels, Argonne, Conference on Safety, Fuels and Core Design, Oct. 1965 ANL 7120

12) R. Haase, Thermodynamik der irreversiblen Prozesse (Dr. D. Steinkopff-Verlag, Darmstadt, 1963)

13) A. B. Lidiard, Mass transfer along a temperature gradient (IAEA Symposium, Vienna, July 1965)
14) N. Oi und J. Takagi, Z. Naturf. 19a (1964) 1331

$\left.{ }^{15}\right)$ K. Wirtz, Phys. Z. 44, 11 (1943) 221

16) A. B. Auskern und J. Belle, J. Nucl. Mat. 3 (1961) 311

17) R. Lindner und F. Schmitz, Z. Naturf. 16a (1961) 1373

18) J. H. Davis und P. E. Novak, ANS Meeting San Francisco Calif. (Dec. 1964)

${ }^{19}$ G. B. Alcock, R. J. Hawkins, A. W. D. Hills und P. MoNamara, Thermodynamics (IAEA Symposium, Vienna, July 1965) 